\title{
L'Encyclopédie Garland des musiques du monde (suite)
}

The Garland Encyclopedia of World Music. New York and London: Garland Publishing, Inc., 1998-2004

Yves Defrance

\section{(2) OpenEdition}

Journals

Édition électronique

URL : http://journals.openedition.org/ethnomusicologie/710

ISSN : 2235-7688

Éditeur

ADEM - Ateliers d'ethnomusicologie

Édition imprimée

Date de publication : 1 janvier 2001

Pagination : 221-229

ISBN : 2-8257-0723-6

ISSN : 1662-372X

\section{Référence électronique}

Yves Defrance, "L'Encyclopédie Garland des musiques du monde (suite) », Cahiers d'ethnomusicologie [En ligne], 13 | 2001, mis en ligne le 09 janvier 2012, consulté le 03 mai 2019. URL : http:// journals.openedition.org/ethnomusicologie/710

Ce document a été généré automatiquement le 3 mai 2019.

Tous droits réservés 


\section{L'Encyclopédie Garland des musiques du monde (suite)}

The Garland Encyclopedia of World Music. New York and London:

Garland Publishing, Inc., 1998-2004

\section{Yves Defrance}

\section{RÉFÉRENCE}

L'Encyclopédie Garland des musiques du monde (suite). The Garland Encyclopedia of World Music. New York and London: Garland Publishing, Inc., 1998-2004, 10 vol Vol. 4: Southeast Asia (Asie du Sud-Est), par Terry E. Miller \& Sean Williams (ed.), 1998. 1024 p. + CD encarté.

Vol. 9: Australia and the Pacific Islands (Australie et îles du Pacifique), par Adrienne L. Kaeppler \& John W. Love (ed.), 1998. 1088 p. + CD encarté.

\section{NOTE DE L'ÉDITEUR}

Ce compte rendu fait suite à celui des volumes 1 et 2 de l'encyclopédie Garland paru l'an dernier dans les Cahiers (Defrance 1999: 199-207)

\section{New York and London: Garland Publishing, Inc., 1998-2004, 10 vol}

L'édition des dix volumes annoncés n'étant pas achevée, les comptes rendus de cette grande encyclopédie ne peuvent se faire qu'au fur et à mesure des livraisons et avec le décalage habituel qu'impose le rythme annuel de notre revue. De plus, les éditions Garland sont elles-mêmes tributaires de l'avancée des manuscrits, ce qui fait que l'ordre 
de parution ne suit pas nécessairement la numérotation des volumes. Après tout, nous savons que telle deuxième symphonie fut composée avant telle première et cela ne gâche en rien notre écoute. La lecture de l'encyclopédie Garland procure, elle aussi, bien des plaisirs: plaisir de la découverte; et personne ne peut prétendre ne rien apprendre dans ces sommes d'informations en tout genre; plaisir de suivre la démonstration d'une réflexion pénétrante, d'une théorie nouvelle, d'une approche originale, d'un concept opératoire; plaisir de lire la formulation de pensées, l'organisation d'analyses, l'approche de terrains, la définition de problématiques dans une dialectique constante entre local et global plaçant l'anecdotique aux côtés de l'universel; plaisir de l'écoute de documents sonores inédits ou d'accès très difficile; plaisir, au fond, de se sentir concerné par des questions a priori fort éloignées de celles que l'on traite sur ses propres terrains.

2 Pour la première fois, un grand nombre de travaux sont regroupés par régions du monde et, bien que nécessairement non exhaustifs, ils offrent un panorama général d'une très grande richesse. Il est vrai que le spécialiste a tendance à guetter, l'œil critique, la livraison de la région qu'il étudie, pour se précipiter sur les coquilles, voire les erreurs. La tentation est grande de s'arrêter sur les petits détails auxquels chacun est en droit de tenir. Quand on se lance dans l'aventure encyclopédique, on en mesure très vite les limites. L'ethnomusicologie, en particulier anglo-saxonne, revêt parfois des allures tentaculaires et l'hyperspécialisation ne prend de sens que dans un contexte de réflexions communes et générales. Gardons à l'esprit que chacun puisse lire l'autre. Du point de vue de l'éditeur, la tentation est tout aussi grande de placer sur le marché des produits bâclés mais financièrement rentables. Les exemples ne manquent pas, hélas, de publications «grand public» truffées d'inexactitudes, de stéréotypes et de fausses vérités. Le compromis entre une encyclopédie "complète» - qui, en supposant que les questions budgétaires soient résolues, mettrait des dizaines d'années à voir le jour - et un objet de vulgarisation par trop réductrice me semble tout à l'honneur de l'éditeur.

3 Avec l'encyclopédie Garland, nous avons un outil d'une bonne fiabilité et d'une remarquable efficacité, tant dans son utilisation que dans la manière de traiter des questions générales. Si «impasses» il y a, l'essentiel reste copieusement documenté. Ce qui importe, à mon sens, tient en la démarche même d'un tel projet. L'intention réside bien dans l'esprit de synthèse et non dans l'accumulation de petits faits. Inévitablement, cela conduit à des choix. Au lecteur d'en mesurer la justesse.

\section{Vol. 4: Southeast Asia (Asie du Sud-Est), par Terry E. Miller \& Sean Williams (ed.), 1998. 1024 p. + CD encarté}

4 Organisé en trois parties inégales, ce volume est conduit par Terry E. Miller et Sean Williams qui, à eux seuls, fournissent près du quart des articles (vue d'ensemble, état des connaissances, thématiques propres, études de cas, etc.). L'introduction pose habilement plus de questions qu'elle ne donne de réponses. Il est vrai que la diversité géographique et culturelle de ce sous-continent asiatique entraîne des différences très prononcées dans les productions musicales. Alors qu'il peut être admis, à la rigueur, de parler de musiques africaines, il semble impossible de regrouper sous un seul terme l'ensemble des musiques de cette vaste région, de surcroît fort peuplée. De plus, comme cela est fréquent, l'organisation politique actuelle ressort, pour une bonne part, d'un découpage colonial 
souvent en contradiction avec des réalités certes plus anciennes, mais au cœur de l'identité et de l'élaboration esthétique de certaines cultures. Coincé entre la Chine, la Birmanie, le Cambodge et le Viêt-nam, le Laos n'est par exemple que le vestige des spoliations de la guerre. A l'inverse, la mise au point d'un répertoire «classique» aux Philippines, semble plus refléter une réaction d'un pays fortement christianisé face aux modèles dominants occidentaux, que le prolongement d'une réelle pratique de cour profondément ancrée dans la culture locale. De même, les influences bouddhiques, indiennes, chinoises et arabes (par le truchement de la conversion à l'islam d'une partie importante des populations entre océans Indien et Pacifique) ne laissent pas d'interroger les observateurs sur les origines de tel instrument, de telle pratique musicale.

Division entre mondes continentaux et mondes insulaires, populations des hautes et des basses terres, particularités régionales, clivages habituels urbain/rural, musiques classique/populaire (avec une distinction subtile entre folk et popular) et réseaux d'influence, conduisent les auteurs à dresser un inventaire de questions «non résolues» qui me semblent très pertinentes. Quelle est l'origine des populations de l'Asie du SudEst? Si telle d'entre elles vient de Yunnan (Chine), qu'est-il advenu des aborigènes, et quelle part ont-ils pris dans la construction de son identité? Cette question de l'identité revient constamment dans les travaux. Et si la «pureté ethnique» n'existe pas, à partir de quand et comment se développe une culture distincte? Concernant la musique, qu'est-ce qui ressort de l'indigène et de l'exogène, voire de l'acculturé? La notion d'authenticité, toute relative, est bien commode pour placer des repères mais ne résiste que très rarement à l'analyse. D'un côté les observateurs extérieurs ont vite tendance à établir des similitudes entre les cultures et les musiques du monde. De l'autre, les érudits locaux insistent peut-être un peu trop sur les caractéristiques qu'ils voient en leur propre culture. Sans doute, sont-ils particulièrement à même de prendre en compte avec finesse les mille et une subtilités qui font vivre leur musique - et l'on sait combien la dimension stylistique compte dans la définition de soi - mais à ne considérer que les à-côtés d'une musique ils en oublieraient les lignes de forces majeures. Et là se pose à nouveau la question des origines. Si l'on admet que les populations des hautes terres, minoritaires et souvent marginalisées, représentent les "premières phases» culturelles en Asie du SudEst, alors il faut considérer toute une panoplie d'instruments de musique comme fondamentaux à cette région: gongs de bronze, guimbardes de bambou et de métal, aérophones à anche libre, orgues à bouche, xylophones de bambou verticaux, etc. Ce qui implique que les ensembles comme le gamelan indonésien, le piphat thaï ou le pinn peat cambodgien se seraient déjà développés à partir des premiers occupants, aujourd'hui montagnards. De même, personne n'est en mesure d'assurer que les instruments de bronze des îles de Bornéo et d'au-delà furent effectivement importés par des migrants venus du continent. Rien ne semble infirmer l'hypothèse d'une transmission par les terres en des temps préhistoriques avant la montée des eaux. L'histoire plus récente nous met également en garde contre des interprétations trop rapides. Les diverses conquêtes militaires qui marquèrent la région au cours des cinq derniers siècles concoururent à l'établissement de cours princières, elles-mêmes pourvues d'ensembles musicaux raffinés. Est-ce à dire que la musique thaï reflète la première phase de la musique cambodgienne? La musique de cour lao fut-elle influencée par la musique cambodgienne du fait que la cour de Luang Phrabang fut instaurée avec l'aide des Khmers? Est-ce seulement après 1767 que les Birmans acquirent des instruments semblables à ceux des Thaïs? La musique 
balinaise actuelle sonne-t-elle comme la musique de cour javanaise du xive siècle, puisque celle-ci fut introduite, du moins partiellement, à Bali?

6 Ces questions des origines dépassent très largement le cadre ethnomusicologique et occupent une bonne partie du débat anthropologique, notamment dans les études linguistiques. Le terrain, très sensible, s'y prête bien et impose même aux chercheurs de telles thématiques. Au point que Terry E. Miller et Sean Williams consacrent encore deux articles de fond dans la deuxième partie aux «Vagues d'influences culturelles», et à «La culture, la politique, la guerre».

7 Mais, dans cette introduction, les auteurs n'en oublient pas l'essentiel et, d'une façon somme toute très pédagogique, nous convient à l'écoute: «How to listen to southeast asian musics». Sont considérées aussi bien les approches sensuelles qu'intellectuelles, avant qu'une présentation générale par régions ne guide le lecteur. Le souci pratique reste constant et les étapes à suivre pour pénétrer l'univers de ces musiques sont tracées de sorte que le néophyte complet ne se décourage pas trop vite. Un ton qui peut parfois irriter l'ethnomusicologue, mais, encore une fois, cette encyclopédie s'ouvre à un large public et il est tout à fait possible d'y trouver son compte en allant directement chercher l'information souhaitée.

8 Limités à quinze, les exemples sonores, d'excellente qualité, privilégient la durée, quatre minutes en moyenne. Les neuf enregistrements réalisés par Terry E. Miller mettent en avant la Thaïlande (4) et le Viêt-nam (3) laissant peu de place à Java (1) ou à Bali (1). Mais n'est-il pas préférable de proposer, comme ici, des pièces rares et diversifiées qui font voler en éclat les stéréotypes? D'autant que certaines régions, ravagées par les guerres et les folies meurtrières au cours du dernier tiers $d u x^{e}$ siècle, ne sont plus toujours en mesure de fournir des exemples vivants de cultures et de pratiques musicales séculaires. Les témoignages et documents sonores d'avant cette sinistre période n'en sont que plus précieux et dégagent une véritable émotion. Idée originale, la première plage présente un montage de trois séquences de chant des grenouilles dans la campagne thaillandaise. Véritable symphonie de la nature, cet environnement sonore prend une signification de réjouissance pour les paysans d'Asie du Sud-Est. Il indique d'abondantes précipitations, garantissant une bonne récolte de riz et annonçant la présence de délicieux poissons, crevettes, insectes et grenouilles. Batraciens que l'on retrouve, avec le crapaud, dans la mythologie indonésienne, elle-même à la base de la pratique musicale (guimbarde enggung, cérémonies du cak, motif du crapaud dans les pièces pour gamelan javanais, etc.). Le chant classique birman kyo, accompagné à la harpe arquée saung, aux cliquettes wa et aux cymbales hsi, est une pure merveille. Enregistré le 23 juin 1994 au village de Binh Son dans la province de Thanh Hoa, Viêt-nam, le magnifique chant dialogué de la minorité Thai Dam montre qu'il est encore possible de faire de belles découvertes. La place manque pour présenter tous ces beaux exemples qui permettront, à l'occasion, de se familiariser avec le pélog, à défaut de slèndro. Laissons à chacun la satisfaction d'une écoute qui, du début à la fin, ravit les oreilles les plus blasées

9 Six articles étoffés exposent quelques problématiques dans la seconde partie. Karl L.Hutterer traite de la préhistoire, Robert Wessing du rapport entre le mode de vie et la culture, dans un titre sans ambiguïté: «Bamboo, Rice, and Water». Deborah Wong, pour la Thaïlande, et René T.A.Lysloff, pour l'Indonésie, présentent des travaux récents sur les musiques populaires dans ces pays industrialisés qui conduisent eux-mêmes des politiques culturelles influentes. Les deux maîtres d'œuvre de ce volume livrent, outre les deux articles cités plus haut, une étude sur l'impact de la modernité sur les musiques 
traditionnelles prenant notamment en compte l'effet des concours, du tourisme, du revival, de l'enseignement, des nouvelles compositions, etc. Autant de thèmes de recherche vraisemblablement voués à un bel avenir et qui commencent timidement à être pris en considération dans les publications de langue française. Intitulée "Cultures musicales et régions», la troisième partie occupe les quatre cinquièmes de l'ouvrage. Vingt-sept monographies de Terry E. Miller, Sean Williams et vingt et un autres chercheurs, sont regroupées géographiquement: - Cultures majoritaires continentales (8): «Introduction»; «Les Khmers»; «La Thaïlande»; «Le Laos»; «La Birmanie»; «La péninsule malaise»; «Le Viêt-nam»; «Singapour». - Peuples minoritaires des hautes terre (5): «Introduction»; «Musique minoritaires du Viêt-nam»; «Musique des minorités des hautes terres de Birmanie, Laos et Thailande»; «Les populations de la péninsule malaise»; «Les Châm des basses terres». - Iles (14): «Introduction; «Sumatra»; «Java»; «Bali»; «Nusa Tenggara Barat» (province d'Indonésie à l'est de Bali); «Nusa Tenggara Timur» (Timor, Sumba, Flores et autres); «Célèbes»; «Moluques»; «Bornéo»; «Les basses terres chrétiennes philippines»; «Musique savante du $\mathrm{xx}^{\mathrm{e}}$ siècle aux Philippines»; «Musique populaire aux Philippines»; «Communautés islamiques au sud des Philippines»; «Peuples des hautes terres aux Philippines».

11 Selon la matière et l'avancée des travaux, la longueur des articles varie d'une page (les Châm) à cent pages (Java). Certains articles relèvent d'études anciennes comme celle Hans Oesch sur les Orang Asli de Malaisie, reprise et complétée par Marina Roseman. D’autres, notamment sur les minorités, donnent les premiers résultats d'investigations récentes. Tout comme dans les premiers volumes de l'encyclopédie, la parole est donnée, autant que faire se peut, à des chercheurs originaires eux-mêmes des régions étudiées. On se réjouit des communications de quatre chercheurs de l'Université des Philippines, qui apportent véritablement de la matière neuve: Arnold Cabalza, Corazon Canave-Dioquino, José Maceda et Ram $-\mathrm{n}$ P. Santos. Trois chercheurs australiens (Christopher Basile, Janet Hoskins et Margaret J.Kartomi), un Indonésien (Endo Suanda) un Japonais (Ruriko Uchida) et un Néerlandais (Robert Wessing) se sont également joints à l'équipe des seize Américains (Amy Catlin, James Chopyak, David Harnish, Karl L. Hutterer, Ward Keeler, Lee Tong Soon, Patricia Marusky, Phong T. Nguyên, Panya Roongrang, Sam-Ang Sam, R. Anderson Sutton, Deborah Wong) eux-mêmes répartis dans une douzaine d'universités, ce qui montre un bel esprit d'ouverture.

12 Par contre, la qualité des photographies noir et blanc, beaucoup trop contrastées, laisse franchement à désirer. Est-ce un problème d'impression? On a du mal à croire que les auteurs n'aient pu fournir de bons documents iconographiques. Ceci nuit franchement à l'ensemble, d'autant que la superbe première de couverture, en couleur, laisse présager d'une édition luxueuse. La cartographie, simple et claire, ignore les reliefs alors que l'on sait combien la dichotomie hautes/basses terres s'avère pertinente dans ces régions. Le lecteur n'a d'ailleurs pas toujours de carte pour suivre une démonstration (ex. Nusa Tenggara Barat: pp. 762-785). Un petit problème matériel déjà rencontré dans les premiers volumes: on reste bien en peine lorsqu'il faut se battre au cutter avec l'emballage plastifié du CD. Celui-ci, trop solidement encarté, risque par la suite de s'égarer, faute de réceptacle approprié! Au demeurant, il eut été très facile, et peu onéreux, d'encarter $2 \mathrm{CD}$ au lieu d'un seul. La matière ne manque pas et les appétits sont vite aiguisés par les descriptions de la troisième partie: descriptions qui, à mon sens, auraient gagné à prendre un peu plus en compte la danse et le théâtre, si imbriqués dans les cultures d'Asie du Sud-Est. Autre point faible, le peu d'intérêt porté pour l'analyse. 
Sauf exception, les textes se contentent du stade énumératif dans des inventaires un peu fastidieux, pas toujours bien écrits et, mise à part l'introduction, plutôt affirmatifs qu'interrogatifs. L'esprit critique semble paralysé par la crainte de commettre une faute déontologique et on ne sent pas toujours la finalité d'une présentation, encore moins son insertion dans une réflexion ethnomusicologique plus globale.

Il reste que cette synthèse, enrichie de travaux inédits sur des populations et des musiques peu ou pas étudiées avant ces vingt dernières années, offre une excellente entrée en matière.

\section{Vol. 9: Australia and the Pacific Islands (Australie et îles du Pacifique), par Adrienne L. Kaeppler \& John W. Love (ed.), 1998. 1088 p. + CD encarté.}

14 Mises à part la filmographie dressée par John W. Love et la présentation très générale d'Adrienne L. Kaeppler, en quatre pages, tous les articles sont rédigés collectivement. Certains mobilisent jusqu'à vingt-cinq plumes. Alors que l'Asie du Sud-Est émane principalement de deux personnes, pas moins de cent soixante-sept chercheurs ont contribué à la rédaction du volume sur l'Océanie. Bien évidemment, les américains, y compris un grand nombre d'ethnommusicologues hawaïens, arrivent en tête. Il y a également de nombreux Australiens, Néo-Zélandais, Européens, trois Japonais, un Chilien, et une vingtaine d'Océaniens ou d'Occidentaux établis en Océanie. Nous avons là, à la fois une bonne variété d'approches et une nécessaire convergence de points de vue. Erudition et qualité de réflexion en bénéficient grandement. Non pas que tout soit parfait, mais il est remarquable de réussir à mobiliser autant de spécialistes sur une région, certes très éparpillée géographiquement mais, somme toute, assez peu peuplée.

Il faut dire que ces terrains étaient pratiquement inexplorés avant 1922, date de la parution d'Argonauts of the Western Pacific, mais qu'ils furent le point de mire d'un grand nombre d'anthropologues par la suite. Les deux célèbres enquêtes de Bronislaw Malinowski mirent brutalement les projecteurs de la recherche internationale sur la Nouvelle-Guinée et les îles Trobriand. Son enseignement à la London School of Economics, puis à Yale (Etats-Unis) contribua à susciter de nouvelles vocations d'enquêteurs, et les ethnomusicologues ne tardèrent pas à leur emboîter le pas. Nombreux sont, en effet, ceux qui inscrivirent dans leur carrière un terrain océanien avant de s'illustrer dans d'autres domaines (Wolfgang Laade, Dieter Christensen, Hugo Zemp, Jean-Michel Beaudet, Artur Simon). Au-delà des clichés occidentaux véhiculés par les récits d'explorateurs depuis le xvi ${ }^{\mathrm{e}}$ siècle, où les visiteurs sont accueillis par des danses de femmes, des colliers de fleurs et autres délicatesses, l'Océanie exerce une séduction puissante auprès des chercheurs. Quoi de plus attirant que d'établir une monographie musicale sur un terrain défini par l'insularité, l'isolement et l'homogénéité de son peuplement? Si l'on a tout lieu de penser qu'une partie des populations océaniennes vinrent d'Asie orientale et d'Asie du Sud-Est, il y a plusieurs milliers d'années, leurs relations avec d'autres continents restèrent longtemps réduites. Ce qui fait qu'elles développèrent par elles-mêmes des systèmes musicaux tout à fait originaux. Les 54 exemples du CD d'accompagnement présentent une palette de prestations individuelles et collectives, vocales et instrumentales, monodiques et polyphoniques, rituelles et récréatives du plus grand intérêt. Presque tous inédits, ces enregistrements de terrain 
apportent une illustration sonore indispensable à la compréhension des langages musicaux de sociétés où la notion de musique d'art - parfois même de musique tout court - est complètement absente. La qualité de certaines prises de son, en particulier celles de John W. Love et Adrienne L. Kaeppler, et ceux de Gerald Florian Messner, est saisissante. Elles plongent l'auditeur dans des univers inhabituels, repoussant très loin les limites de la définition du champ d'investigation des études ethnomusicologiques.

Quatre parties rythment l'ouvrage: «Introduction à l'Océanie et sa musique»; «Concepts dans la musique d'Océanie»; «Les peuples d'Océanie et leur musique»; et «Ressources et outils de recherche».

Pour les Occidentaux, l'Océanie renferme des sociétés qui, ayons l'honnêteté de le reconnaître, focalisèrent sur elles l'image la plus négative qui soit. La violence morale et physique avec laquelle furent traités les habitants des terres conquises par les Européens était encore, hélas, bien visible durant la seconde moitié $d u x^{e}$ siècle. L'ethnocentrisme manifesté par exemple à l'encontre des Aborigènes d'Australie ou des Kanaks de Nouvelle Calédonie, qui n'obtinrent le droit de vote que dans les années 1960, donne aujourd'hui des frissons. Curieusement, deux clichés contradictoires s'opposent. Celui du bon sauvage, vivant en paix sur une île que d'aucuns ont cru pouvoir identifier au Paradis terrestre; et celui du méchant sauvage, rebelle et insaisissable, que l'on renonce à «éduquer». Ils sont accompagnés d'autant de clichés musicaux qui vont de la guitare hawaïenne à l'ukulele valorisé au cinéma dans les mains d'une Marylin Monroe, en passant par les «curiosités» comme les flûtes nasales et le très exotique didjeridoo qui, revanche de l'histoire, envahit aujourd'hui le moindre trottoir des capitales européennes. Cette distance culturelle d'avec les peuples d'Océanie attira particulièrement les chercheurs occidentaux, conscients qu'ils étaient des enjeux dans une redéfinition de l'altérité. Quelques régions d'Océanie, comme la Papouasie, sont considérées, aujourd'hui encore, parmi les plus difficiles d'accès et les moins bien connues de la planète. Peu d'ethnologues s'y aventurent et nos informations sur les cultures et les musiques de leurs habitants restent très incomplètes.

De tels faits, et bien d'autres encore, sont rappelés dans une première partie à caractère historique: découverte de l'autre, découverte de soi, migrations musicales. D'une soixantaine de pages, le dernier chapitre évoque les importants mouvements de population qui marquèrent cette vaste région et en modifièrent très sensiblement les équilibres. Certaines îles reçurent peu de colons, d'autres en subirent les assauts par vagues successives. Pour Hawaï, l'Australie, les Fidji et la Nouvelle Calédonie, les habitants originaux sont devenus minoritaires. La Polynésie française risque de connaître le même sort puisque l'État français encourage ses fonctionnaires métropolitains à y prendre leur retraite, source de revenus au troisième rang dans l'économie locale. Il n'empêche que les musiques des groupes ethniques établis en Océanie au cours des trois derniers siècles ont aussi droit à considération. Sont passés en revue les Chinois, les Grecs, les Hmongs (originaires du Laos et des camps de Thaïlande), les Irlandais, Ecossais, Italiens, Latinoaméricains, Caribéens, Indiens, Japonais, Porto-Ricains, Philippins, Américains.

19 La seconde partie, à mon sens la plus intéressante et la plus achevée, mène une série de réflexions qui dépassent très largement le cadre géographique et prennent souvent une dimension universelle. Leur simple énumération donne une idée de la variété des approches, toutes fortement influencées par le courant de l'anthropologie culturelle. Une première série intitulée «Musique et société» traite des thèmes suivants: la musique populaire, musique et absorption de drogues, musique et religion, musique et politique, 
musique et théâtre, musique et sexe social (gender), musique et éducation. La seconde série, dite «Les nombreuses dimensions de la musique» aborde les questions de: comprendre la musique, comprendre la danse, la langue parlée et/ou chantée dans les arrangements musicaux, les accoutrements dans la prestation musicale, les procédés de composition, les instruments de musique.

Forte de 460 pages, la troisième grande partie connaît une subdivision géographique entre l'Australie, la Nouvelle Guinée, la Mélanésie, la Micronésie et la Polynésie. Chacun de ces chapitres commence par une présentation générale des musiques et des danses, puis décrit les particularités musicales et chorégraphiques d'aires plus réduites. La juxtaposition de telles monographies apporte un complément utile pour une vision générale de toutes ces musiques, précisant leurs singularités et la complexité de leur imbrication dans les cultures auxquelles elles se rattachent.

21 La grande variété des populations étudiées, l'isolement de nombreuses d'entre elles avant l'arrivée des ethnologues et la qualité des méthodes d'observation participante mises en œuvre par les scientifiques firent de l'Océanie une sorte de laboratoire idéal pour les chercheurs. Ces conditions exceptionnelles permirent aux ethnomusicologues d'enrichir considérablement le corpus des savoirs sur des questions essentielles: organologie, systèmes musicaux, polyphonie vocale et instrumentale, etc. L'apport des terrains océaniens se mesure peut-être encore plus lorsqu'il est question, non pas d'isoler des enregistrements à des fins d'analyse comparative, mais de situer les pratiques musicales, voire linguistiques et tout simplement sonores, dans leurs contextes culturels. La lecture de ce volume 9 de l'encyclopédie Garland incite à replacer l'homme au centre des préoccupations. Elle pousse le lecteur à s'interroger sur ses propres terrains, voire même à reconsidérer des notions parfois un peu vite oubliées. Si les théories fonctionnalistes, élaborées par Malinowski sur le terrain et à partir de son expérience, se révélèrent par la suite peu fécondes pour l'ethnologie en général, il apparaît que, pour les ethnomusicologues, ce souci de la «totalité» ouvrit de belles perspectives de recherche, difficilement imaginables auparavant. Ce gros volume en donne la plus parfaite démonstration. Malheureusement, les mêmes remarques que précédemment sont à formuler quant à la qualité des photographies illustrant l'ouvrage.

Aux références données à la fin de chaque article sont ajoutés systématiquement en fin des ouvrages glossaire, bibliographie, discographie, filmographie et index. Ces listes suivent encore l'ordre alphabétique en plaçant le nom avant le prénom, habitude universitaire prise depuis des siècles en Europe, et c'est tant mieux. Je dis encore, car je vois poindre aux Etats-Unis l'affreuse façon de placer le prénom avant le nom dans les bibliographies, et qui a pour effet néfaste de ralentir la consultation. A cela se généralise une nouvelle mode: organiser une bibliographie par date de parution et non plus par auteur. Quand les deux manies se combinent vous êtes sûrs d'y perdre des cheveux. Gageons que cette hypocrite correction politique ne contamine pas les esprits sains. 